Research Article

\title{
Effects of Conjugate Heat Transfer on Steady MHD Mixed Convective Heat Transfer Flow over a Thin Vertical Plate Embedded in a Porous Medium with High Porosity
}

\begin{abstract}
Ahmet Kaya
Department of Mechanical Engineering, Kahramanmaras Sutcu Imam University, 46100 Kahramanmaras, Turkey

Correspondence should be addressed to Ahmet Kaya, ekaya38@gmail.com

Received 8 May 2012; Accepted 4 June 2012

Academic Editor: Serge Prudhomme

Copyright (C) 2012 Ahmet Kaya. This is an open access article distributed under the Creative Commons Attribution License, which permits unrestricted use, distribution, and reproduction in any medium, provided the original work is properly cited.

This study investigates mixed convection heat transfer about a thin vertical plate in the presence of magneto and conjugate heat transfer effects in the porous medium with high porosity. The fluid is assumed to be incompressible and dense. The nonlinear coupled parabolic partial differential equations governing the flow are transformed into the nonsimilar boundary layer equations, which are then solved numerically using the Keller box method. The effects of the conjugate heat transfer parameter $p$, the porous medium parameter $k_{1}$, the Forchheimer parameter $F^{*}$, the mixed convection parameter $\mathrm{Ri}$, the magnetic parameter $\mathrm{Mn}$, and the electric field parameter $E_{1}$ on the velocity and temperature profiles as well as on the local skin friction and local heat transfer are presented and analyzed. The validity of the methodology and analysis is checked by comparing the results obtained for some specific cases with those available in the literature.
\end{abstract}

\section{Introduction}

Thermal buoyancy-induced flow and convective heat transfer in fluid-saturated porous media have been the subject of numerous publications. This interest in the subject stems from various engineering applications in geothermal reservoirs, petroleum industries, transpiration cooling, storage of radioactive nuclear waste materials, separation processes in chemical industries, building thermal insulation, and solar heating systems [1]. Kaviany [2], Pop and Ingham [3], Ingham and Pop [4, 5], and Nield and Bejan [6] have made comprehensive reviews of the studies of heat transfer in relation to the above applications. 
The conjugate heat transfer problem, in which the coupled heat transfer processes between the solid body (conduction mechanisms) and the fluid flow (convection mechanisms) are considered simultaneously, has been investigated by several researchers for with and without porous medium. Miyamoto et al. [7] studied two-dimensional conjugate heat transfer problems of free convection from a vertical flat plate with a uniform temperature or a uniform heat flux at the outsidem surface of the plate. Sparrow and Chyu [8] investigated the conjugate problem for a vertical plate fin with various heat transfer coefficients under forced convection. They assumed that the heat conduction in the fin was to be one-dimensional. Char et al. [9] employed the cubic spline collocation numerical method to analyze the conjugate heat transfer in the laminar boundary layer on a continuous, moving plate. Wang [10] studied the thermo-fluid-dynamic field resulting from the coupling of wall conduction with laminar mixed convection heat transfer of micropolar fluids along a vertical flat plate. Pop et al. [11] presented a detailed numerical study of the conjugate mixed convection flow along a vertical flat plate. Chang [12] presented a numerical analysis of the flow and heat transfer characteristics of mixed convection in a micropolar fluid flowing along a vertical flat plate with conduction effects. He assumed that the heat conduction in the wall was only in the transversal direction. Mamun et al. [13] investigated the heat generation effect on natural convection flow along and conduction inside a vertical flat plate. Hsiao and Hsu [14] studied a conjugate mixed convection heat transfer problem of a second-grade viscoelastic fluid past a horizontal flat-plate fin.

For porous medium, Pop and $\mathrm{Na}$ [15] reported a numerical study of the steady conjugate free convection over a vertical slender, hollow circular cylinder with the inner surface at a constant temperature and embedded in a porous medium. Vaszi et al. [16] investigated two-dimensional conjugate free convection from an inclined flat plate in a semiinfinite porous medium under the boundary-layer approximation. Méndez et al. [17] studied steady state heat transfer characteristics of a thin vertical strip with internal heat generation placed in a porous medium. Saeid [18] studied steady conjugate natural convection in two-dimensional vertical porous layer sandwiched between two equal-thickness walls. The Darcy model was used in the mathematical formulation for the porous layer and finite volume method was used to solve the dimensionless governing equations. Vaszi et al. [19] investigated two-dimensional conjugate free convection in a porous medium from a vertical plate fin. Hossain et al. [20] investigated conjugate free convection along a vertical cylindrical fin in a non-Newtonian fluid-saturated porous medium. The boundary layer equations based on the power law model appropriate for the Darcy flows were solved numerically. This article illustrates the effect of wall conduction on non-Darcy MHD mixed convection flow over a thin vertical plate embedded in a porous medium with high porosity. The boundary layer equations governing the flow are reduced to local nonsimilarity equations which are solved using the implicit finite difference method (Keller box). Variation in the fluid-solid interfacial temperature distribution, the local skin friction and local heat transfer parameters as well as the velocity and temperature profiles are presented to highlight the influence of the wall conduction, porous medium parameter, buoyancy, electric field, and magnetic effects parameters.

This study considers two-dimensional, steady, laminar, non-Darcy, incompressible electrically conducting fluid flow over a thin vertical plate of length $L$ and finite thickness $b(L \gg b)$ embedded in a porous medium. The physical model and coordinate system are shown in Figure 1. Far above/below the surfaces of the thin vertical plate, the velocity and the temperature of the free stream are $u_{\infty}$ and $T_{\infty}$, respectively. The temperature of the inside surface of the plate is maintained at a constant temperature of $T_{0}$, where $T_{0}>T_{\infty}$. The flow 
region is exposed under uniform transverse magnetic fields $\vec{B}=\left(0, B_{0}, 0\right)$ and uniform electric field $\vec{E}=\left(0,0,-E_{0}\right)$ [21]. Magnetic and electric fields are known from Maxwell's equation that $\nabla \cdot \vec{B}=0$ and $\nabla \times \vec{E}=0$. When magnetic field is not so strong then electric field and magnetic field obey Ohm's law $\vec{J}=\sigma(\vec{E}+\vec{q} \times \vec{B})$, where $\vec{J}$ is the Joule current, $\sigma$ is the magnetic permeability and $\vec{q}$ is the fluid velocity. It is assumed that magnetic Reynolds number of the fluid is small so that induced magnetic field and Hall effect may be neglected and takes into account of magnetic field effect as well as electric field in momentum boundary layer equation [21]. The porous medium is assumed to be transparent and in thermal equilibrium with the fluid. Both the fluid and the porous medium are opaque for self-emitted thermal radiation. The properties of the fluid and the porous media, such as viscosity, thermal conductivity, specific heat, and permeability, are assumed to be constant. The porous medium is considered to be homogeneous and isotropic (i.e., uniform with a constant porosity and permeability).

\section{Analysis}

The governing equations for this investigation are based on the usual boundary-layer equations modified to include the porous medium effects, the magnetic and electric field effects, and the thermal buoyancy effects. These equations (with the Boussinesq and nonDarcy approximations) can be written as (see Chamkha et al. [1] and Chamkha [22])

$$
\begin{gathered}
\frac{\partial u}{\partial x}+\frac{\partial v}{\partial y}=0 \\
\frac{1}{\varepsilon^{2}}\left[u \frac{\partial u}{\partial x}+v \frac{\partial u}{\partial y}\right]=\frac{v}{\varepsilon} \frac{\partial^{2} u}{\partial y^{2}}+\frac{\sigma}{\rho}\left[E_{0} B_{0}-B_{0}^{2}\left(u-u_{\infty}\right)\right] \\
-\frac{v}{K}\left(u-u_{\infty}\right)-F\left(u^{2}-u_{\infty}^{2}\right)+g \beta\left(T-T_{\infty}\right) \\
u \frac{\partial T}{\partial x}+v \frac{\partial T}{\partial y}=\frac{k_{e}}{\rho c_{p}}\left(\frac{\partial^{2} T}{\partial y^{2}}\right) .
\end{gathered}
$$

The above equations are called Brinkman-Forchheimer-extended-Darcy equations [23]. Here $u$ and $v$ are the velocity components in the $x$ and $y$ directions, respectively; $v$ is the kinematic viscosity; $g$ is the acceleration due to gravity; $k_{e}$ is the effective thermal conductivity of porous medium; $\rho$ is the density of the fluid; $\beta$ is the coefficient of thermal expansion; $T$ is the temperature of the fluid inside the thermal boundary layer; $K$ is the permeability of the porous medium; $F$ is the empirical constant (Forchheimer number) in the second-order resistance and setting $F=0$ in (2.2), the equation is then reduced to the Darcy's law. The third and fourth terms on the right hand side of (2.2) stand for the first-order (Darcy) resistance and second-order porous inertia resistance, respectively [21]. The thermal dispersion effect is minimal when the thermal diffusivity $\left(k_{e} / \rho c_{p}\right)$ of the porous matrix is of the same order of magnitude as that of the working fluid [1]. 


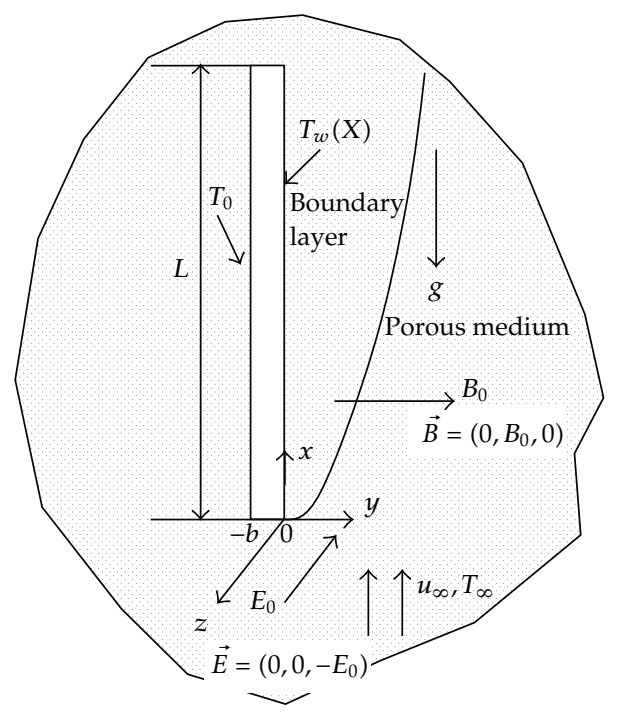

Figure 1: The schematic of the problem.

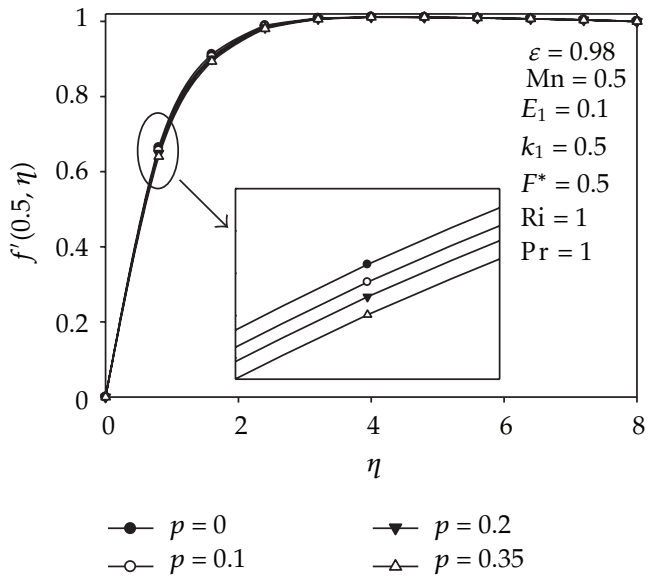

(a)

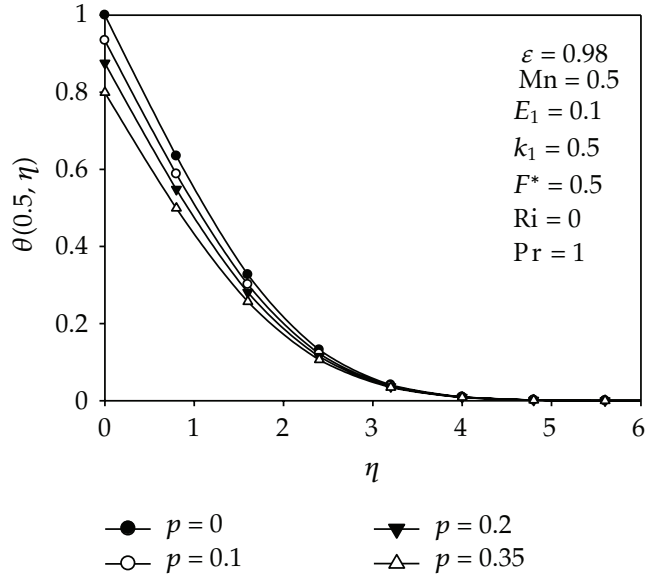

(b)

Figure 2: Dimensionless velocity (a) and temperature (b) profiles for different $p$ while $\varepsilon=0.98, \operatorname{Pr}=1.0$, $\mathrm{Ri}=1, \mathrm{Mn}=1, E_{1}=0.1, k_{1}=0.5, F^{*}=0.5$ and $\xi=0.5$.

The appropriate boundary conditions for the velocity and temperature of this problem are

$$
\begin{gathered}
x=0, \quad y>0, \quad T=T_{\infty}, \quad u=u_{\infty}, \\
x>0, \quad y=0, \quad T=T_{w}(x), \quad u=0, \quad v=0, \\
y \longrightarrow \infty, \quad T \longrightarrow T_{\infty}, \quad u \longrightarrow u_{\infty},
\end{gathered}
$$


where subscripts $w$ and $\infty$ refer to the wall and the boundary layer edge, respectively. In addition, $T_{w}(x)$ is the surface temperature of the plate, which is not known a priori.

One objective of the current study is to predict the surface temperature of the plate $T_{w}(x)$. Therefore, an additional governing equation is required for the plate based on the simplification that the plate steadily transfers its heat to the surrounding fluid. Since the thickness of the plate, $b$, is small compared with its length, $L$, the axial conduction term in the heat conduction equation of the thin plate can be omitted [24, 25]. The governing equation for the temperature distribution within the plate is given by

$$
\left.\frac{d^{2} T}{d y^{2}}\right|_{s}=0 ; \quad 0 \leq x \leq L ;-b<y \leq 0
$$

The boundary conditions for the wall of plate are given

$$
\text { At } y=-b, \quad T_{s}=T_{0},
$$

$$
\text { At the interface }(y=0), \quad T_{s}=T(x, 0):-\left.k_{s} \frac{d T}{d y}\right|_{y=0, s}=-\left.k_{f} \frac{\partial T(x, 0)}{\partial y}\right|_{y=0, f},
$$

where $k_{s}$ and $k_{f}$ are the thermal conductivity of the plate and the fluid, respectively. The boundary conditions given in $(2.6 \mathrm{~b})$ state the physical requirements that the temperature and heat flux of the plate must be continuous across the solid-fluid interface. From (2.5) and (2.6a), (2.6b), the temperature distribution $T_{w}$ at the interface is shown to be

$$
T_{w}(x)=T(x, 0)=b \frac{k_{f}}{k_{s}} \frac{\partial T(x, 0)}{\partial y}+T_{0} .
$$

To seek a solution, the following dimensionless variables are introduced:

$$
\begin{gathered}
\xi(x)=\frac{x}{L}, \quad \psi(x, y)=\left(v u_{\infty} x\right)^{1 / 2} f(\xi, \eta), \\
\eta=y\left(\frac{u_{\infty}}{v x}\right)^{1 / 2}, \quad \theta=\frac{T-T_{\infty}}{T_{0}-T_{\infty}},
\end{gathered}
$$

where $\psi(x, y)$ is the free stream function that satisfies (2.1) with $u=\partial \psi / \partial y$ and $v=-\partial \psi / \partial x$. In terms of these new variables, the velocity components can be expressed as

$$
\begin{gathered}
u=u_{\infty} f^{\prime}, \\
v=-\frac{\left(v u_{\infty} x\right)^{\frac{1}{2}}}{x}\left\{\frac{1}{2} f+\xi \frac{\partial f}{\partial \xi}-\frac{\eta}{2} f^{\prime}\right\} .
\end{gathered}
$$




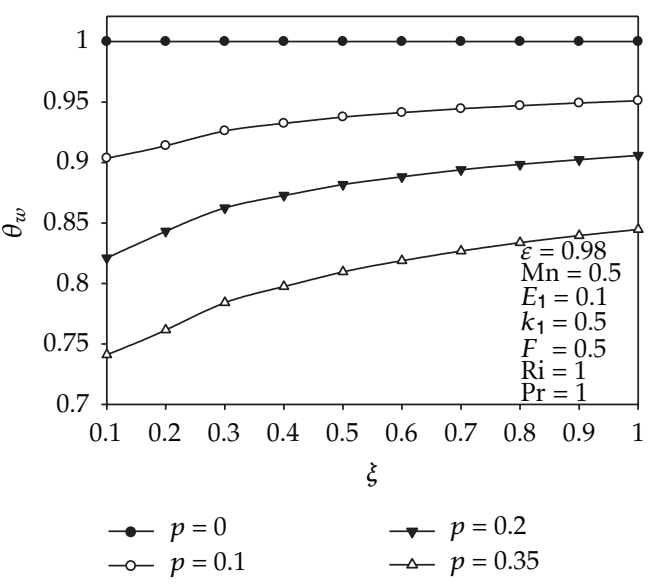

(a)

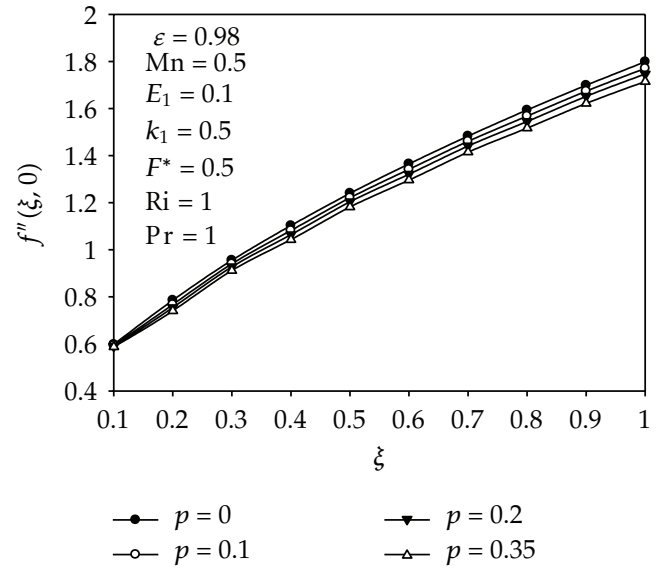

(b)

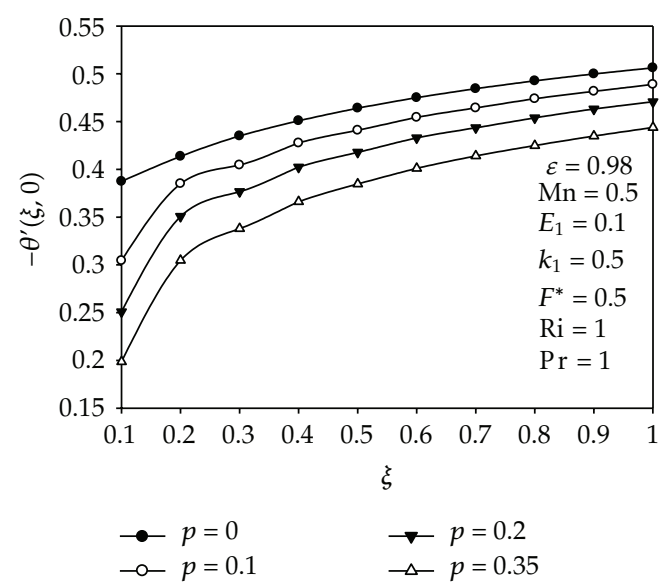

(c)

Figure 3: Effect of conjugate heat transfer parameter $p$ on the dimensionless interfacial temperature (a), local skin friction (b), and local heat transfer (c) parameters against the streamwise distance $\xi$ while $\varepsilon=$ $0.98, \operatorname{Pr}=1.0, \operatorname{Ri}=1, \mathrm{Mn}=1, E_{1}=0.1, k_{1}=0.5$, and $F^{*}=0.5$.

The transformed momentum and energy equations together with the boundary conditions, (2.2), (2.3), and (2.5), can be written as

$$
\begin{aligned}
& \frac{1}{\varepsilon} f^{\prime \prime \prime}+ \frac{1}{2 \varepsilon^{2}} f f^{\prime \prime}+\operatorname{Mn} \xi\left[E_{1}-\left(f^{\prime}-1\right)\right]-k_{1} \xi\left(f^{\prime}-1\right)-F^{*} \xi\left[\left(f^{\prime}\right)^{2}-1\right] \\
&+\operatorname{Ri} \xi \theta=\frac{\xi}{\varepsilon^{2}}\left(f^{\prime} \frac{\partial f^{\prime}}{\partial \xi}-f^{\prime \prime} \frac{\partial f}{\partial \xi}\right) \\
& \frac{1}{\operatorname{Pr}} \theta^{\prime \prime}+\frac{1}{2} f \theta^{\prime}=\xi\left(f^{\prime} \frac{\partial \theta}{\partial \xi}-\theta^{\prime} \frac{\partial f}{\partial \xi}\right)
\end{aligned}
$$




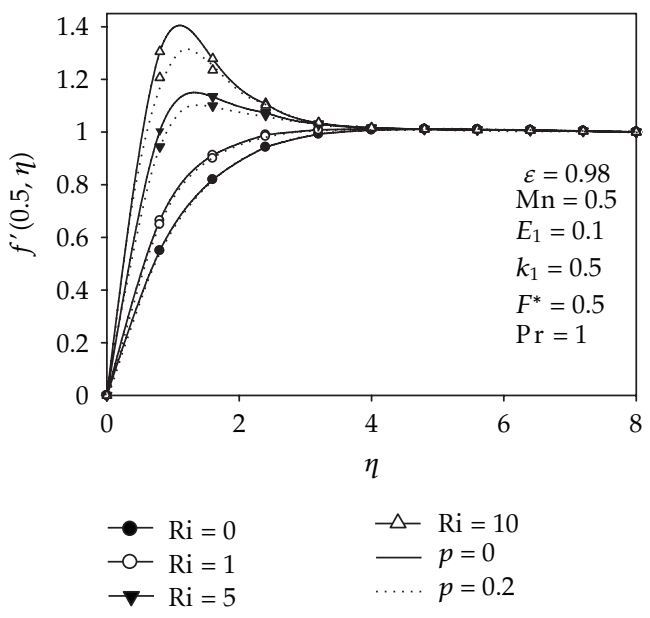

(a)

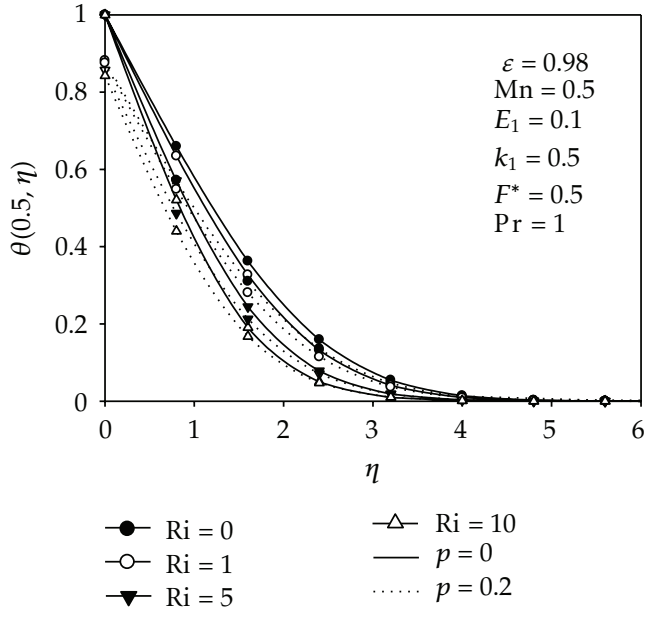

(b)

Figure 4: Dimensionless velocity (a) and temperature (b) profiles for different Ri while $\varepsilon=0.98, \operatorname{Pr}=1.0$, $\mathrm{Mn}=1, E_{1}=0.1, k_{1}=0.5, F^{*}=0.5$, and $\xi=0.5$.

with the boundary conditions:

$$
\begin{gathered}
f(\xi, 0)+2 \xi \frac{\partial f}{\partial \xi}=0, \quad f^{\prime}(\xi, 0)=0, \quad \theta(\xi, 0)-1=p \xi^{-1 / 2} \theta^{\prime}(\xi, 0), \\
f^{\prime}(\xi, \infty)=1, \quad \theta(\xi, \infty)=0,
\end{gathered}
$$

where $p=\left(k_{f} / k_{s}\right)(b / L) \operatorname{Re}_{L}^{1 / 2}$ is the conjugate heat transfer parameter. It should be noticed that for the limiting case of $p=0$, the thermal boundary condition in (2.12) on the wall becomes isothermal. Hence, the magnitude of $p$ determines the importance of the wall heat conduction effect [25]. defined as:

The corresponding dimensionless groups that appeared in the governing equations

$$
\begin{aligned}
\mathrm{Mn} & =\frac{\mathrm{Ha}}{\mathrm{Re}}, \quad \mathrm{Ha}=\frac{\sigma B_{0}^{2} L^{2}}{\mu}, \quad E_{1}=\frac{E}{\mathrm{Re}^{\prime}} \\
E & =\frac{E_{0}}{B_{0} v / L}, \quad k_{1}=\frac{v L}{K u_{\infty}}, \quad F^{*}=F L, \\
\mathrm{Ri} & =\frac{\mathrm{Gr}_{L}}{\operatorname{Re}_{L}}, \quad \mathrm{Gr}_{L}=\frac{g \beta\left(T_{w}-T_{\infty}\right) K L}{v^{2}}, \quad \operatorname{Re}_{L}=\frac{u_{\infty} L}{v}, \quad \operatorname{Pr}=\frac{\mu c_{p}}{k}=\frac{v}{\alpha},
\end{aligned}
$$

where $\mathrm{Mn}$ is the magnetic parameter; Ha is the Hartman number; $E_{1}$ is the electric field parameter; $k_{1}$ is the porous medium parameter; $F^{*}$ is the Forchheimer parameter expressing the relative importance of the inertia effect. $\mathrm{Ri}$ is the Richardson number, which measures the relative importance of free to forced convection. $\mathrm{Ri}=0$ corresponds to the case of purely forced convection condition. $\mathrm{Ri} \rightarrow \infty$ corresponds to the case of purely free convection 


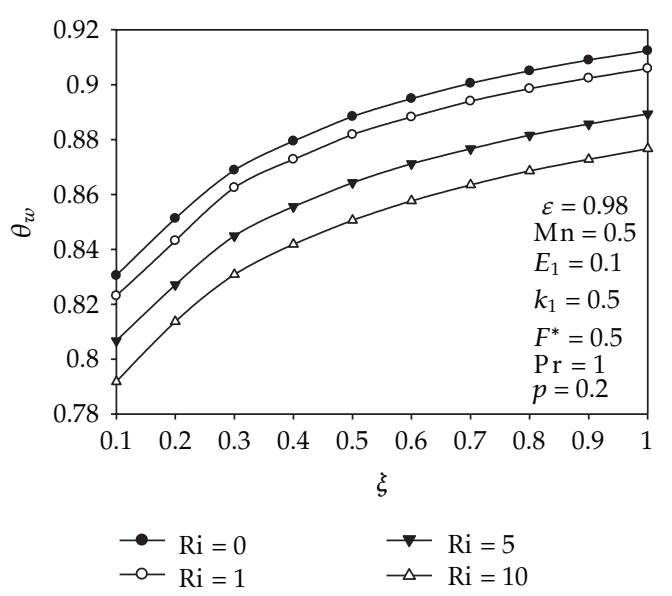

(a)

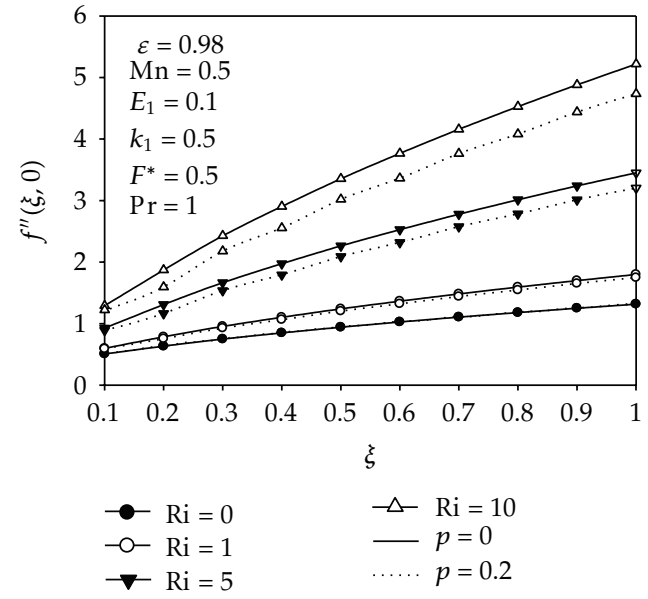

(b)

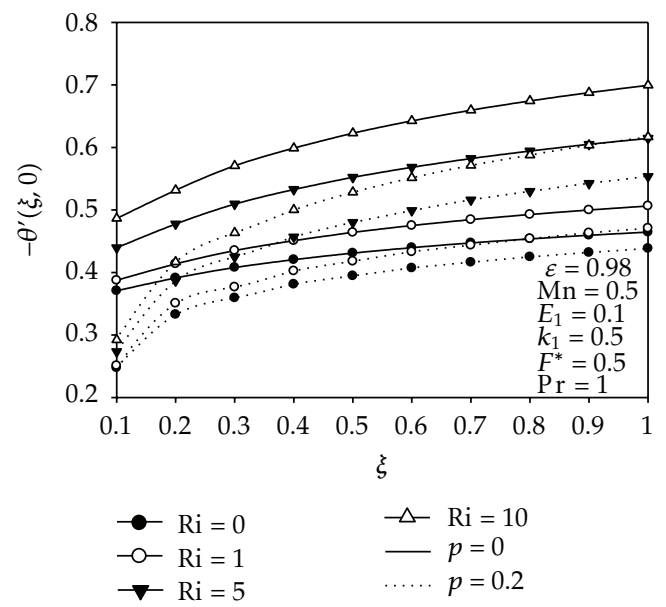

(c)

Figure 5: Effect of Ri on the dimensionless interfacial temperature (a), local skin friction (b), and local heat transfer (c) parameters against the streamwise distance $\xi$ while $\varepsilon=0.98, \operatorname{Pr}=1.0, \mathrm{Mn}=1, E_{1}=0.1, k_{1}=0.5$, and $F^{*}=0.5$.

condition. It is noted that $\mathrm{Ri}$ is not the function of $x . \mathrm{Gr}_{L}$ is the average Grashof number; $\operatorname{Re}_{L}$ is the average Reynolds number; Pr is the Prandtl number.

From the definition of the dimensionless wall temperature, it can be shown that

$$
\theta_{w}=\frac{T_{w}-T_{\infty}}{T_{0}-T_{\infty}}
$$

\section{Numerical Solution}

The system of transformed equations under the boundary conditions, Equations (2.11), (2.12), has been solved numerically using the Keller box scheme, which is proved to be an efficient and accurate finite-difference scheme [30]. Readers are referred to Cebeci and 


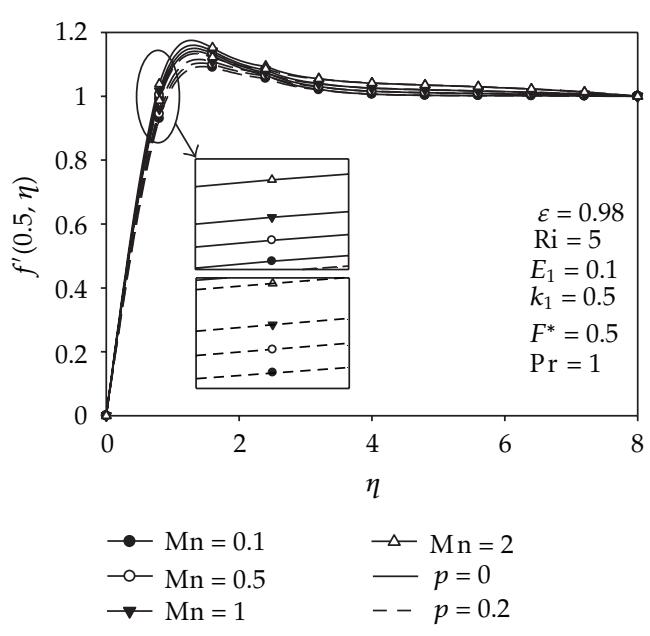

(a)

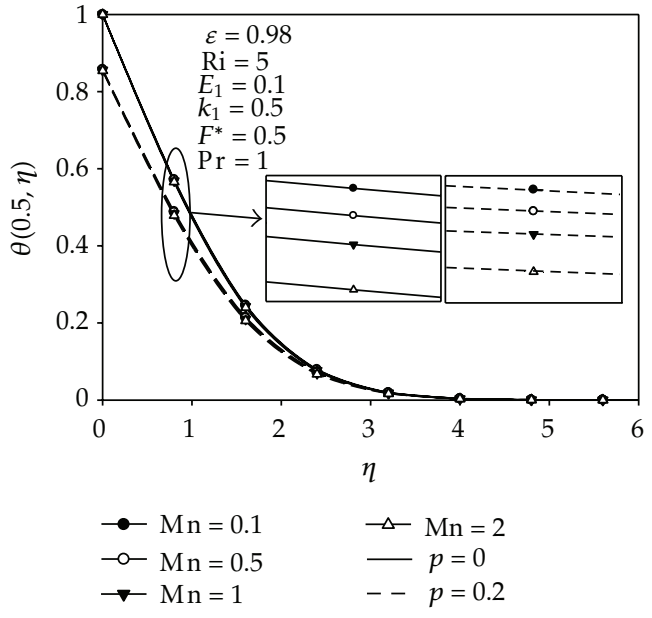

(b)

Figure 6: Dimensionless velocity (a) and temperature (b) profiles for different $\mathrm{Mn}$ while $\varepsilon=0.98, \operatorname{Pr}=1.0$, $\mathrm{Ri}=5, E_{1}=0.1, k_{1}=0.5, F^{*}=0.5$ and $\xi=0.5$.

Bradshaw [30] for the details of the numerical methods. This is a very popular implicit scheme, which demonstrates the ability to solve systems of differential equations of any order as well as featuring second-order accuracy (which can be realized with arbitrary non-uniform spacing), allowing very rapid $x$ or $\xi$ variations [31].

A set of nonlinear finite-difference algebraic equations derived are then solved by using the Newton quazi-linearization method. The same methodology as that followed by Hossain et al. [20] is followed. Therefore, for the finite-difference forms of the equations, the reader is referred to Takhar and Beg [31] for the brevity of the paper.

In the calculations, a uniform grid of the step size 0.01 in the $\eta$-direction and a nonuniform grid in the $\xi$-direction with a starting step size 0.001 and an increase of 0.1 times the previous step size were found to be satisfactory in obtaining sufficient accuracy within a tolerance better than $10^{-6}$ in nearly all cases. The value of $\eta_{\infty}=16$ is shown to satisfy the velocity to reach the relevant stream velocity.

In order to verify the accuracy of the present method, the present results were compared with those of Kuznetsov and Nield [26] (Table 1), Chamkha et al. [27], and Aydin and Kaya [28] (Table 2) and Aydin and Kaya [29] (Table 3). The comparison is found to be in good agreement, as shown in Tables 1, 2, and 3, respectively.

\section{Results and Discussion}

The aim of this study was to investigate the flow and heat transfer characteristics for the nonDarcy mhd mixed convection flow over a thin vertical plate with wall conduction effect. The following ranges of the main parameters are considered: conjugate heat transfer parameter $p=0.0,0.1,0.2$, and 0.35 ; mixed convection parameter $\mathrm{Ri}=0.0,1.0,5.0$, and 10.0; magnetic interaction parameter $\mathrm{Mn}=0.0,0.5,1.0$, and 2.0; electric field parameter $E_{1}=0.1,0.5,1.0$, and 2.0; porous medium parameter $k_{1}=0.1,0.5,1.0$ and 2; inertia parameter $F^{*}=0.1,0.5,1.0$, and 2.0; $\operatorname{Pr}=1.0$ and $\varepsilon=0.98$. Hence, the numerical computations were performed. 


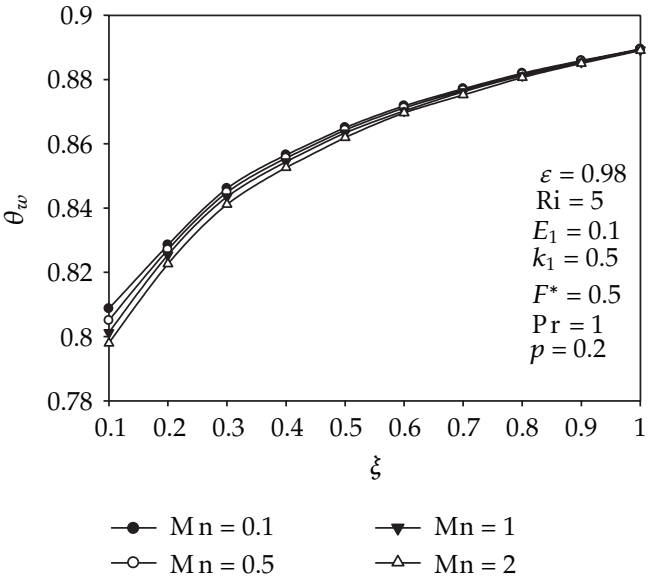

(a)

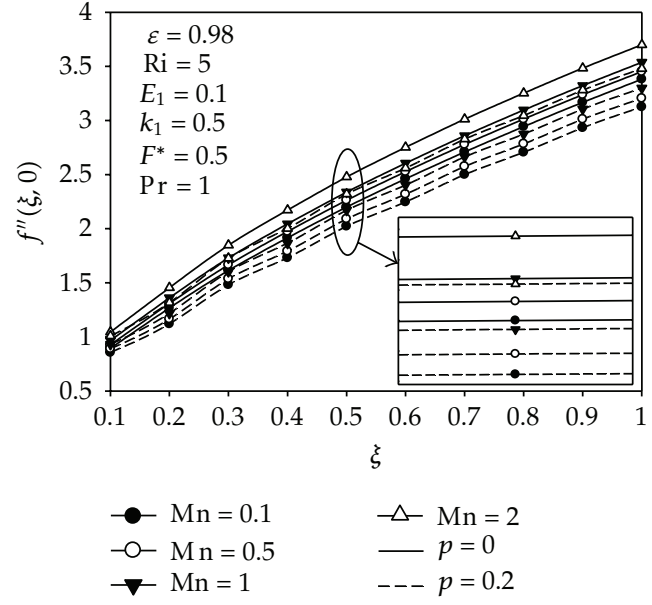

(b)

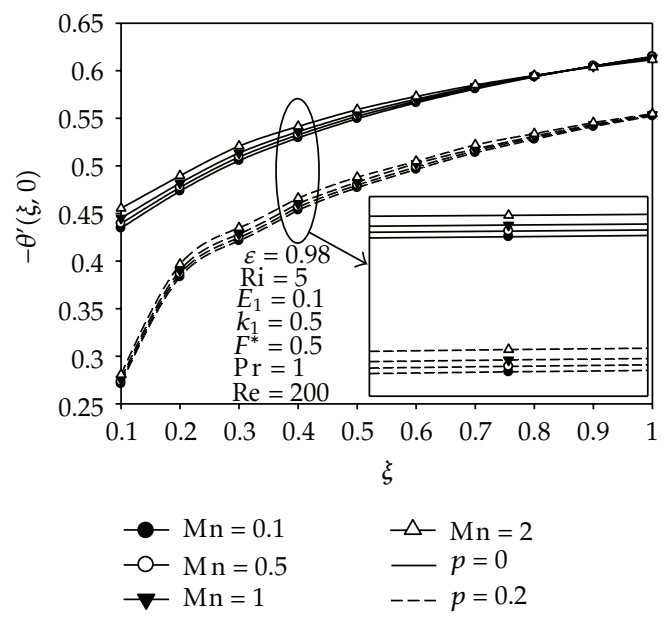

(c)

Figure 7: Effect of Mn on the dimensionless interfacial temperature (a), local skin friction (b), and local heat transfer (c) parameters against the streamwise distance $\xi$ while $\varepsilon=0.98, \operatorname{Pr}=1.0, \operatorname{Ri}=5, E_{1}=0.1$, $k_{1}=0.5$, and $F^{*}=0.5$.

The combined effects of $p, \mathrm{Ri}, \mathrm{Mn}, E_{1}, k_{1}$, and $F^{*}$ on the momentum and heat transfer are analyzed. The Richardson number, Ri represents a measure of the effect of the buoyancy in comparison with that of the inertia of the external forced or free stream flow on the heat and fluid flow. Outside the mixed convection region, either the pure forced convection or the free convection analysis can be used to describe accurately the flow or the temperature field. Forced convection is the dominant mode of transport when $\mathrm{Ri} \rightarrow 0$, whereas free convection is the dominant mode when $\mathrm{Ri} \rightarrow \infty$. Buoyancy forces can enhance the surface heat transfer rate when they assist the forced convection [32].

The effect of conjugate heat transfer parameter $p$ on the velocity (a) and temperature (b) profiles within the boundary layer with $\mathrm{Ri}=1.0, \mathrm{Mn}=0.5, E_{1}=0.1, k_{1}=0.5, F^{*}=0.5$, and $\operatorname{Pr}=1.0$ is shown in Figures 2(a) and 2(b), respectively. The increasing of the conjugate heat transfer parameter decreases velocity and temperature gradients at the wall. A lower 


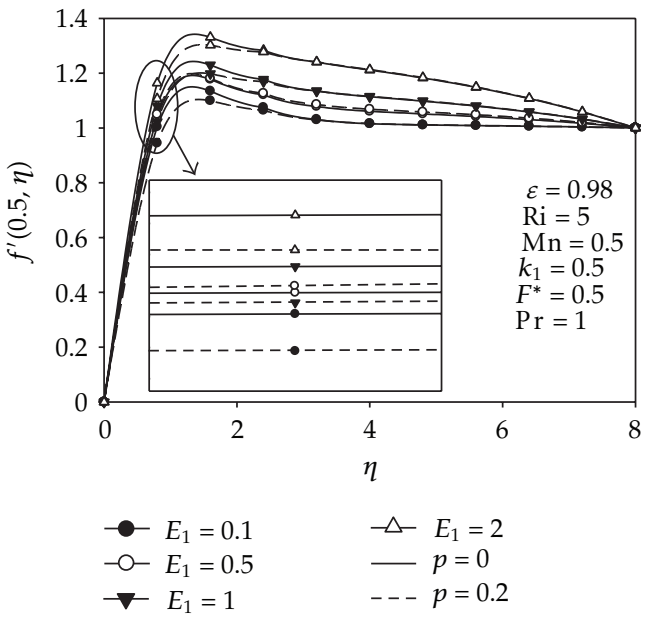

(a)

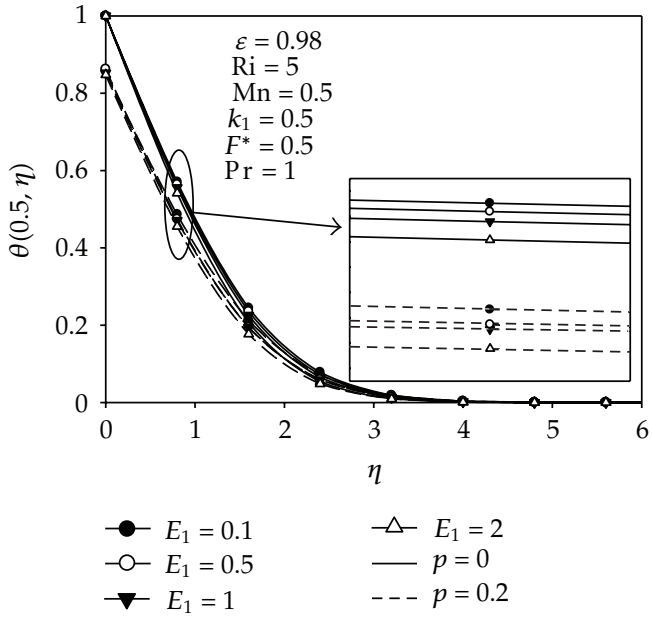

(b)

Figure 8: Dimensionless velocity (a) and temperature (b) profiles for different $E_{1}$ while $\varepsilon=0.98, \operatorname{Pr}=1.0$, $\mathrm{Ri}=5, \mathrm{Mn}=0.5, k_{1}=0.5, F^{*}=0.5$, and $\xi=0.5$.

Table 1: Comparison of the values $-\theta^{\prime}(0,0)$ for various values $\operatorname{Pr}$ at $\mathrm{Mn}=0.0, E_{1}=0.0, \varepsilon=1.0, F^{*}=0.0$, $\mathrm{Ri}=0.0, k_{1}=1.0$, and $p=0.0$.

\begin{tabular}{lcc}
\hline $\operatorname{Pr}$ & Kuznetsov and Nield [26] & Present study \\
\hline 0.1 & 0.158 & 0.148 \\
1 & 0.332 & 0.332 \\
5 & 0.570 & 0.577 \\
10 & 0.730 & 0.728 \\
20 & 0.910 & 0.918 \\
30 & 1.050 & 1.055 \\
40 & 1.150 & 1.155 \\
50 & 1.245 & 1.244 \\
60 & 1.320 & 1.321 \\
70 & 1.390 & 1.390 \\
80 & 1.450 & 1.452 \\
90 & 1.510 & 1.514 \\
100 & 1.570 & 1.573 \\
\hline
\end{tabular}

wall conductance $k_{s}$ or higher convective cooling effect due to greater $k_{f}$ increases the value of $p$ as well as causes greater temperature difference between the two surfaces of the plate. The temperature at the solid-fluid interface is reduced since the temperature at the outside surface of the plate is kept constant.

The variation of the interfacial temperature, the local skin friction, and the local heat transfer parameters for different values of $p$ with $\xi$ are shown in Figures 3(a), 3(b), and 3(c), respectively where $\mathrm{Ri}=1.0, \mathrm{Mn}=0.5, E_{1}=0.1, k_{1}=0.5, F^{*}=0.5$, and $\operatorname{Pr}=1.0$. It can be seen that the temperature of the fluid on the wall increases with $\xi$ for a given value of $p$ (Figure 3(a)). Comparing with isothermal wall $(p=0)$, an increase in the conjugate heat transfer parameter, $p$, causes a reduction in the interfacial temperature. This is because an increased value of $p$ corresponds to a lower wall conductance $k_{s}$ and promotes a greater surface temperature 


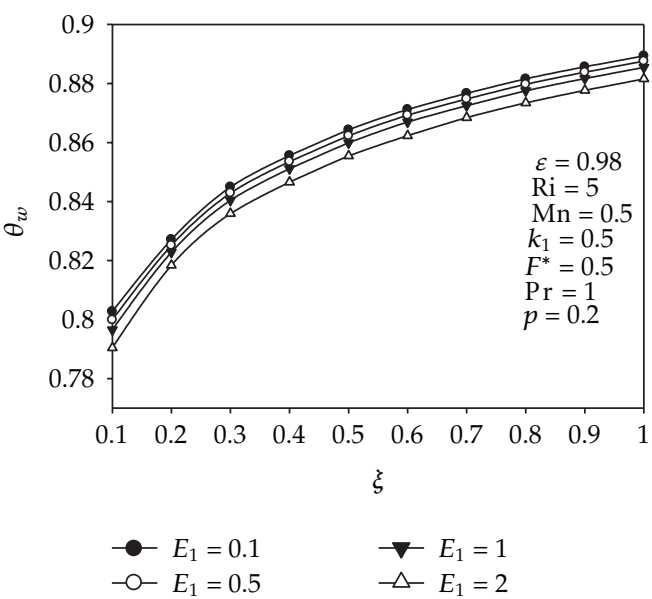

(a)

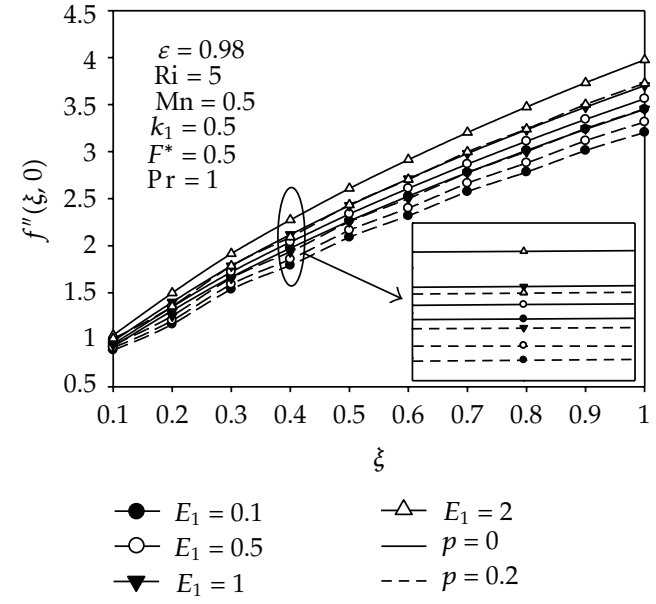

(b)

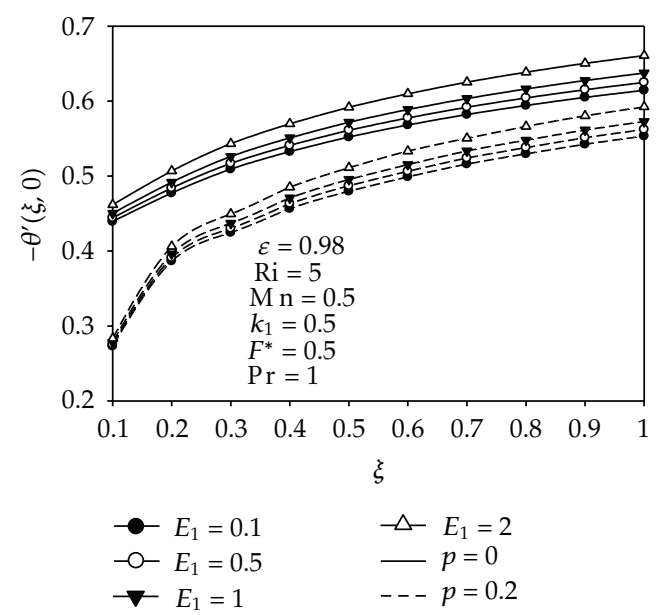

(c)

Figure 9: Effect of $E_{1}$ on the dimensionless interfacial temperature (a), local skin friction (b), and local heat transfer (c) parameters against the streamwise distance $\xi$ while $\varepsilon=0.98, \operatorname{Pr}=1.0, \mathrm{Ri}=5, \mathrm{Mn}=0.5, k_{1}=0.5$, and $F^{*}=0.5$.

Table 2: Comparison of the values $-\theta^{\prime}(\xi, 0)$ for various values $\xi$ with $\operatorname{Pr}=0.7, \mathrm{Mn}=1.0, \operatorname{Ri}=1.0, \varepsilon=1.0$, $F^{*}=0.0, k_{1}=0.0$, and $p=0.0$.

\begin{tabular}{lccc}
\hline$\xi$ & Chamkha et al. [27] & Aydin and Kaya [28] & Present study \\
\hline 0.0 & 0.293 & 0.293 & 0.293 \\
0.01 & 0.322 & 0.330 & 0.330 \\
0.02 & 0.325 & 0.350 & 0.350 \\
0.03 & 0.363 & 0.367 & 0.367 \\
0.04 & 0.380 & 0.379 & 0.379 \\
0.05 & 0.390 & 0.390 & 0.390 \\
0.06 & 0.400 & 0.400 & 0.400 \\
0.07 & 0.409 & 0.408 & 0.408 \\
0.08 & 0.410 & 0.415 & 0.415 \\
\hline
\end{tabular}




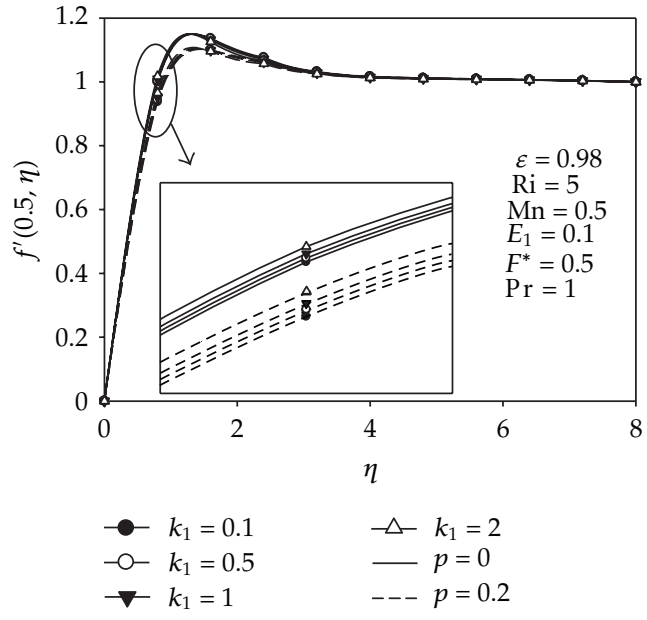

(a)

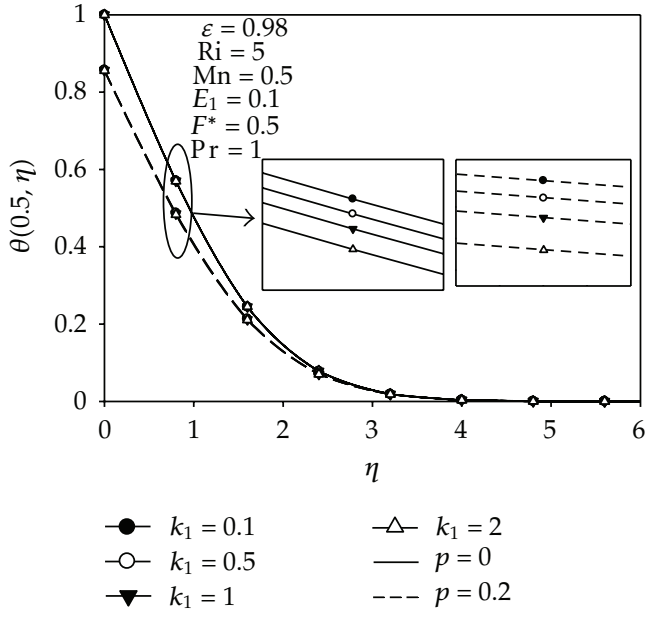

(b)

Figure 10: Dimensionless velocity (a) and temperature (b) profiles for different $k_{1}$ while $\varepsilon=0.98, \operatorname{Pr}=1.0$, $\mathrm{Ri}=5, \mathrm{Mn}=0.5, E_{1}=0.1, F^{*}=0.5$ and $\xi=0.5$.

Table 3: Comparison of the values $-\theta^{\prime}(\xi, 0)$ for various values $\xi$ and $\varepsilon$ with $\operatorname{Pr}=1, F^{*}=0.5, \mathrm{Mn}=0.0, E_{1}=$ $0.0, k_{1}=1.0, \mathrm{Ri}=0.0$, and $p=0.0$.

\begin{tabular}{lcccccc}
\hline & \multicolumn{2}{c}{$\varepsilon=0.5$} & \multicolumn{2}{c}{$\varepsilon=0.75$} & \multicolumn{2}{c}{$\varepsilon=1.0$} \\
$\xi$ & $\begin{array}{c}\text { Aydin and } \\
\text { Kaya [29] }\end{array}$ & Present study & $\begin{array}{c}\text { Aydin and } \\
\text { Kaya [29] }\end{array}$ & Present study & $\begin{array}{c}\text { Aydin and } \\
\text { Kaya [29] }\end{array}$ & Present study \\
\hline 0.0 & 0.367 & 0.367 & 0.346 & 0.346 & 0.332 & 0.332 \\
0.01 & 0.368 & 0.368 & 0.350 & 0.350 & 0.338 & 0.338 \\
0.02 & 0.369 & 0.369 & 0.353 & 0.353 & 0.343 & 0.343 \\
0.03 & 0.371 & 0.371 & 0.355 & 0.355 & 0.348 & 0.348 \\
0.04 & 0.372 & 0.372 & 0.358 & 0.358 & 0.352 & 0.352 \\
0.05 & 0.374 & 0.374 & 0.361 & 0.361 & 0.356 & 0.356 \\
0.06 & 0.375 & 0.375 & 0.364 & 0.364 & 0.360 & 0.360 \\
0.07 & 0.376 & 0.376 & 0.366 & 0.366 & 0.364 & 0.364 \\
0.08 & 0.377 & 0.377 & 0.369 & 0.369 & 0.368 & 0.368 \\
\hline
\end{tabular}

variations (Figure 3(a)). The increases value of $p$ increases the momentum and thermal boundary layer thickness and therefore the local skin friction and the local heat transfer parameters decrease as shown in Figures 3(b) and 3(c). Also, increasing $\xi$ increases the interfacial temperature, the local skin friction, and the local heat transfer parameters (Figures $3(a), 3(b)$, and 3(c)). Similar results were found in the literture $[33,34]$.

Figure 4 shows the dimensionless velocity (a) and temperature (b) profiles inside the boundary layer for different values of the buoyancy parameter Ri for the cases of isothermal plate $(p=0)$ and nonisothermal plate $(p>0)$. The increasing of Ri increases velocity and temperature gradients at the wall. And also, increasing the conjugate heat transfer parameter decreases the velocity and temperature profiles in the boundary layer. 


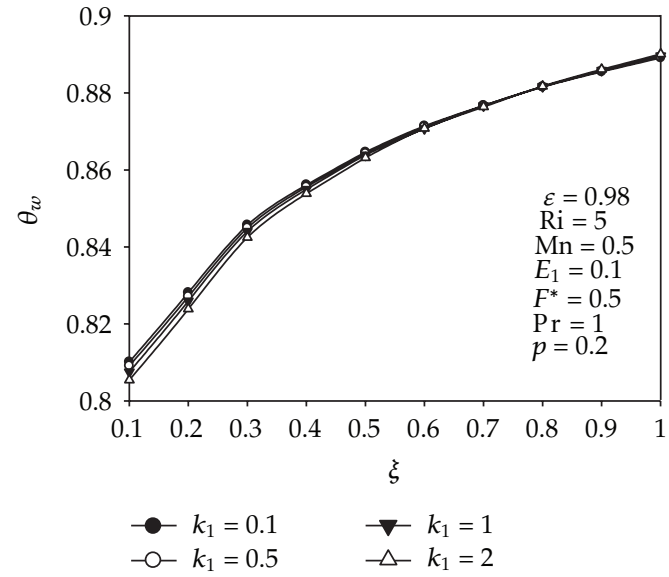

(a)

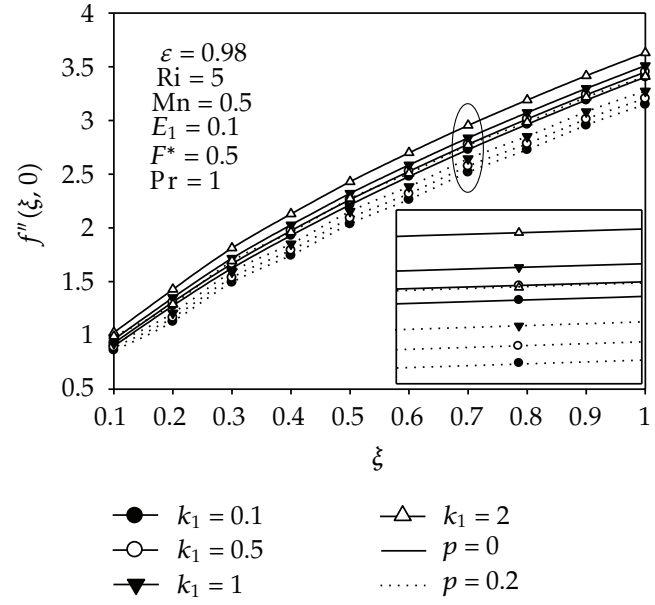

(b)

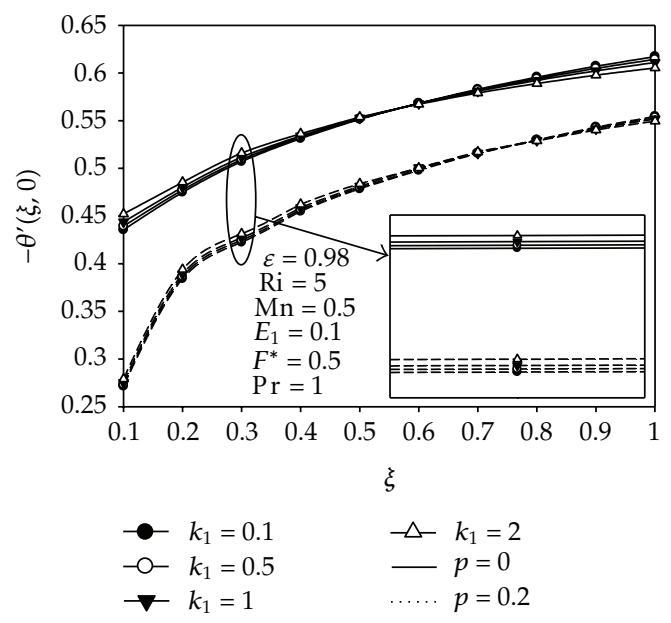

(c)

Figure 11: Effect of $k_{1}$ on the dimensionless interfacial temperature (a), local skin friction (b), and local heat transfer (c) parameters against the streamwise distance $\xi$ while $\varepsilon=0.98, \operatorname{Pr}=1.0, \operatorname{Ri}=5, \mathrm{Mn}=0.5$, $E_{1}=0.1$, and $F^{*}=0.5$.

Figure 5 shows the variation of the dimensionless interfacial temperature distributions (a), the local skin friction (b), and the local heat transfer (c) parameters in the boundary layer. It can be seen that as the values of $\xi$ increases, the interfacial temperature rises. Compared with the limiting case of $\mathrm{Ri}=0.0$, an increase in the value of $\mathrm{Ri}$ gives rise to a reduced interfacial temperature since a greater value of Ri indicates a greater buoyancy effect, which increases the convection cooling effect and hence reduces the wall temperature (Figure 5(a)). Figure $5(\mathrm{~b})$ illustrates the effect of the buoyancy force on the local skin friction factor for $p=0$ (solid lines) and 0.2 (dashed lines). It is observed that the local skin friction parameter increases with the buoyancy effect. The reason for this is that an increase in the buoyancy effect in mixed convection flow leads to an acceleration of the fluid flow, which increases the local skin friction parameter. Additionally, the higher the value of the buoyancy effect, the more the sensitivity of the wall conduction effects influences the skin friction factor. In 


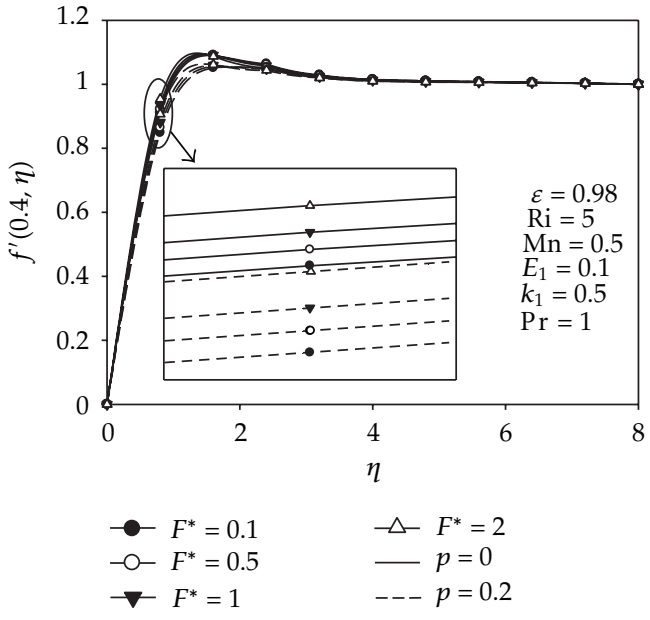

(a)

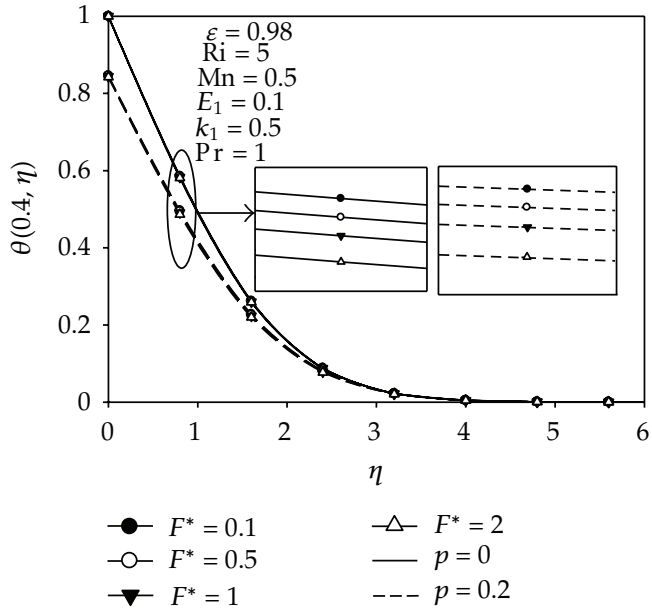

(b)

Figure 12: Dimensionless velocity (a) and temperature (b) profiles for different $F^{*}$ while $\varepsilon=0.98, \operatorname{Pr}=1.0$, $\mathrm{Ri}=5, \mathrm{Mn}=0.5, E_{1}=0.1, k_{1}=0.5$, and $\xi=0.4$.

Figure 5(c), the effect of the buoyancy force on the local heat transfer parameter is illustrated. It is noted that as the value of the Ri increases, the local heat transfer parameter also increases, both for the case of an isothermal plate $(p=0)$ and a nonisothermal plate $(p>0)$. This is because an increased buoyancy effect generates a greater buoyancy force, which increases the fluid velocity and hence raises the local heat transfer parameter [33].

Figure 6 shows the dimensionless velocity (a) and temperature (b) profiles inside the boundary layer for different values of the magnetic parameter $\mathrm{Mn}$ for the cases of isothermal plate $(p=0)$ and nonisothermal plate $(p>0.0)$. The increasing of the magnetic parameter Mn increases velocity and temperature gradients at the wall due to magnetic field effects on external flow field. As mentioned above, increasing the conjugate heat transfer parameter $p$ decreases velocity and temperature gradients at the wall.

The variation of the dimensionless interfacial temperature distributions, the local skin friction, and the local heat transfer parameters in the boundary layer for different magnetic parameter are shown in Figures 7(a), 7(b), and 7(c), respectively. Increasing the magnetic parameter Mn decreases the interfacial temperature (Figure 7(a)). The local skin friction and the local heat transfer parameters with different values of Mn for isothermal plate $(p=0)$ and nonisothermal plate $(p=0.2)$ are illustrated in Figures 7(b) and 7(c). The magnetic force aiding the flow and increases the local skin friction (i.e., shear stress) and the local heat transfer (i.e., heat transfer rate) parameters at the wall.

The dimensionless velocity (a) and temperature (b) profiles inside the boundary layer for different values of the electric field parameter $E_{1}$ for the cases of isothermal plate $(p=0)$ and nonisothermal plate $(p>0.0)$ are illustrated in Figure 8. Increasing of the electric field parameter $E_{1}$ decreases the momentum and thermal boundary layers thickness (Figures 8(a) and $8(\mathrm{~b}))$ because Lorentz force arising due to electric field acts as an accelerating force in reducing the frictional resistance [21]. Therefore, increasing the velocity and temperature gradients at wall increases the local skin friction and local heat transfer parameters as shown in Figures 9(b) and 9(c). Also increasing the electric field parameter $E_{1}$ decreases the interfacial temperature (Figure 9(a)). 


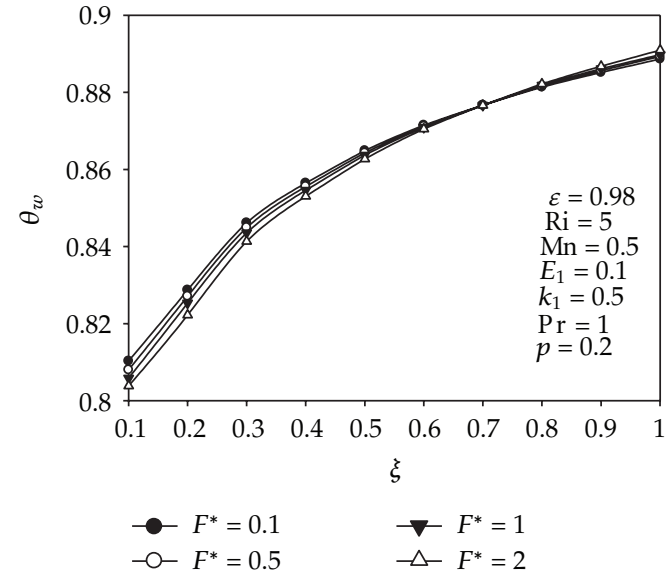

(a)

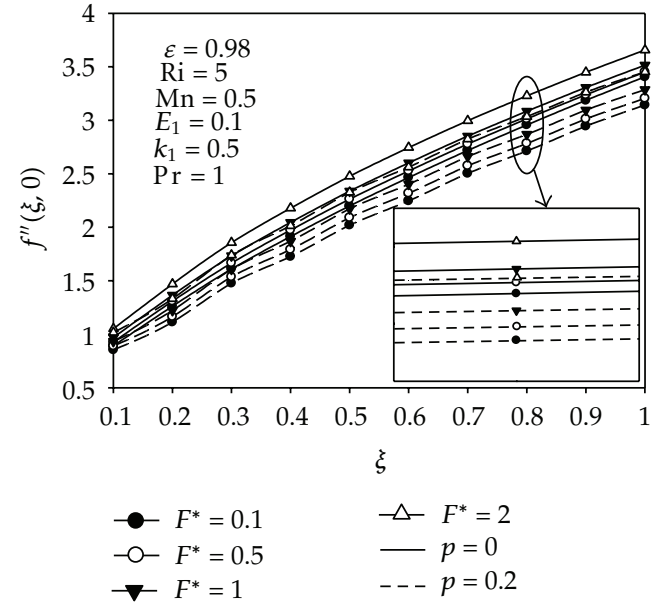

(b)

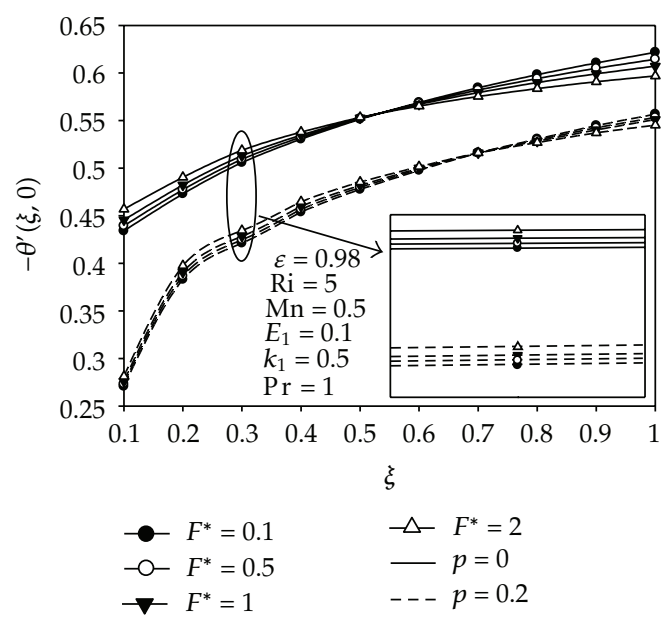

(c)

Figure 13: Effect of $F^{*}$ on the dimensionless interfacial temperature (a), local skin friction (b) and local heat transfer (c) parameters against the streamwise distance $\xi$ while $\varepsilon=0.98, \operatorname{Pr}=1.0, \operatorname{Ri}=5, \mathrm{Mn}=0.5$, $E_{1}=0.1, k_{1}=0.5$.

The increasing of the porous medium parameter $k_{1}$ decreases momentum and thermal boundary layer thickness and increases velocity and temperature profiles for both isothermal $(p=0.0)$ and nonisothermal $(p=0.2)$ plate as shown in Figure 10. Increasing the porous medium parameter decreases the interfacial temperature (Figure 11(a)) and increases local skin friction (Figure 11(b)) and local heat transfer (Figure 11(c)) parameters. The increasing the porous medium parameter $k_{1}$ increases the temperature gradients at wall for $0.1<\xi<$ 0.55 (as expected from (2.9) and as observed in Aydin and Kaya [29] and Kumari and Nath [35]) whereas decreases the temperature gradient at wall for $0.55<\xi<1.0$.

Figure 12 shows the effect of drag (inertia) coefficient of porous medium (or Forchheimer parameter) $F^{*}$ on the dimensionless velocity and temperature profiles at $\xi=0.4$. From this figure it is observed that the effect of drag coefficient $F^{*}$ is to decrease the velocity 
profile, the temperature profile (Figure 12), and the interfacial temperature (Figure 13(a)) and increases local skin friction (Figure 13(b)) and local heat transfer (Figure 13(c)) parameters.

\title{
5. Conclusions
}

This study has analyzed the effect of wall conduction on the non-Darcy MHD mixed convection flow with high porosity. The nonlinear formulation governing equations and their associated boundary conditions have been obtained and solved using the nonsimilarity transform and the finite difference method (Keller box), respectively. The influences of the conjugate heat transfer parameter, mixed convection parameter, magnetic interaction, electric field parameter, porous medium parameter and inertia parameter on the solidliquid interfacial temperature distribution, the local skin friction, and the local heat transfer parameters have been systematically examined. From the present numerical investigation, the following conclusions can be drawn:

(1) An increase in the conjugate heat transfer parameter decreases the velocity and the temperature gradient and therefore decreases the dimensionless interfacial temperature distribution, the local skin friction, and the local heat transfer parameters.

(2) An increase in the magnetic, electric field and buoyancy parameters increases the local skin friction and local heat transfer parameters and decreases the dimensionless interfacial temperature distributions. Increasing $M n, E_{1}$, and $\mathrm{Ri}$ decreases the velocity and temperature gradients at wall for nonisothermal cylinder (i.e., $p>0$ ).

(3) An increase in the porous medium parameter $k_{1}$ and Forchheimer parameter $F^{*}$ increases the local skin friction and local heat transfer parameters and decreases the dimensionless interfacial temperature distributions.

\section{Nomenclature}

\author{
$B_{0}$ : Magnetic field strength \\ $c_{p}$ : Specific heat of the convective fluid \\ $E_{1}$ : $\quad$ Electric field parameter \\ $f:$ Dimensionless stream function \\ $F^{*}$ : Forchheimer parameter \\ Gr: Grashof number \\ Ha: Hartman number \\ $k_{e}$ : Effective thermal conductivity of porous medium \\ $K$ : $\quad$ Porous medium permeability, $\mathrm{m}^{2}$ \\ $k_{1}$ : Porous medium parameter \\ L: Plate length \\ Mn: Magnetic parameter, $M=\mathrm{Ha} / \mathrm{Re}$ \\ Pr: Prandtl number \\ Re: Reynolds number \\ T: $\quad$ Temperature \\ $u, v$ : Velocities in $x$ and $y$ directions, respectively \\ $x, y$ : Coordinates in horizontal and vertical directions, respectively.
}




\section{Greek Symbols}

$\sigma$ : Magnetic permeability

$\beta$ : Coefficient of thermal expansion

$\eta$ : Pseudosimilarity variable, $y \mathrm{Re}_{x}^{1 / 2} / x$

$\xi:$ Nonsimilarity variable, $x / L$

$\rho$ : Fluid density

$\mu$ : Dynamic viscosity

$v$ : Kinematic viscosity

$\theta$ : Dimensionless temperature profile in (2.8).

\section{Subscripts}

s: Solid

w: Wall

$\infty$ : Free stream.

\section{References}

[1] A. J. Chamkha, C. Issa, and K. Khanafer, "Natural convection from an inclined plate embedded in a variable porosity porous medium due to solar radiation," International Journal of Thermal Sciences, vol. 41, no. 1, pp. 73-81, 2002.

[2] M. Kaviany, Principles of Heat Transfer in Porous Media, Springer, New York, NY, USA, 1995.

[3] I. Pop and and D. B. Ingham, Convective Heat Transfer, Pergamon, Amsterdam, The Netherland, 2001.

[4] D. B. Ingham and I. Pop and, Transport Phenomena in Porous Media. I, Pergamon, New York, NY, USA, 1998.

[5] D. B. Ingham and I. Pop and, Transport Phenomena in Porous Media. II, Pergamon, New York, NY, USA, 2002.

[6] D. A. Nield and A. Bejan, Convection in Porous Media, Springer, New York, NY, USA, 1999.

[7] M. Miyamoto, J. Sumikawa, T. Akiyoshi, and T. Nakamura, "Effects of axial heat conduction in a vertical flat plate on free convection heat transfer," International Journal of Heat and Mass Transfer, vol. 23, no. 11, pp. 1545-1553, 1980.

[8] E. M. Sparrow and M. K. Chyu, "Conjugated forced convection-conduction analysis of heat transfer in a plate fin," ASME Journal of Heat Transfer, vol. 104, pp. 204-206, 1982.

[9] M. I. Char, C. K. Chen, and J. W. Cleaver, "Conjugate forced convection heat transfer from a continuous, moving flat sheet," International Journal of Heat and Fluid Flow, vol. 11, no. 3, pp. 257-261, 1990.

[10] T. Y. Wang, "The coupling of conduction with mixed convection of micropolar fluids past a vertical flat plate," International Communications in Heat and Mass Transfer, vol. 25, no. 8, pp. 1075-1084, 1998.

[11] I. Pop, D. B. Ingham, and Y. Yuan, "Mixed convective conjugate heat transfer from a vertical flat plate," Zeitschrift fur Angewandte Mathematik und Mechanik, vol. 76, no. 5, pp. 281-289, 1996.

[12] C. L. Chang, "Numerical simulation of micropolar fluid flow along a flat plate with wall conduction and buoyancy effects," Journal of Physics D, vol. 39, no. 6, article 019, pp. 1132-1140, 2006.

[13] A. A. Mamun, Z. R. Chowdhury, M. A. Azim, and M. A. Maleque, "Conjugate heat transfer for a vertical flat plate with heat generation effect," Nonlinear Analysis. Modelling and Controlling, vol. 13, no. 2, pp. 213-223, 2008.

[14] K. L. Hsiao and C. H. Hsu, "Conjugate heat transfer of mixed convection for viscoelastic fluid past a horizontal flat-plate fin," Applied Thermal Engineering, vol. 29, no. 1, pp. 28-36, 2009.

[15] I. Pop and T. Y. Na, "Conjugate free convection over a vertical slender hollow cylinder embedded in a porous medium," Heat and Mass Transfer, vol. 36, no. 5, pp. 375-379, 2000.

[16] A. Z. Vaszi, D. B. Ingham, D. Lesnic, D. Munslow, and I. Pop, "Conjugate free convection from a slightly inclined plate embedded in a porous medium," Zeitschrift fur Angewandte Mathematik und Mechanik, vol. 81, no. 7, pp. 465-479, 2001. 
[17] F. Méndez, C. Treviño, I. Pop, and A. Liñán, "Conjugate free convection along a thin vertical plate with internal nonuniform heat generation in a porous medium," Heat and Mass Transfer, vol. 38, no. 7-8, pp. 631-638, 2002.

[18] N. H. Saeid, "Conjugate natural convection in a vertical porous layer sandwiched by finite thickness walls," International Communications in Heat and Mass Transfer, vol. 34, no. 2, pp. 210-216, 2007.

[19] A. Z. Vaszi, L. Elliott, D. B. Ingham, and I. Pop, "Conjugate free convection from a vertical plate fin with a rounded tip embedded in a porous medium," International Journal of Heat and Mass Transfer, vol. 47, no. 12-13, pp. 2785-2794, 2004.

[20] M. A. Hossain, A. Nakayama, and I. Pop, “Conjugate free convection of non-Newtonian fluids about a vertical cylindrical fin in porous media," Heat and Mass Transfer, vol. 30, no. 3, pp. 149-153, 1995.

[21] D. Pal and H. Mondal, "Hydromagnetic non-Darcy flow and heat transfer over a stretching sheet in the presence of thermal radiation and Ohmic dissipation," Communications in Nonlinear Science and Numerical Simulation, vol. 15, no. 5, pp. 1197-1209, 2010.

[22] A. J. Chamkha, "Hydromagnetic natural convection from an isothermal inclined surface adjacent to a thermally stratified porous medium," International Journal of Engineering Science, vol. 35, no. 10-11, pp. 975-986, 1997.

[23] G. Lauriat and R. Ghafir, "Forced convective heat transfer in porous media," in Handbook of Porous Media, K. Vafai and H. Hadim, Eds., pp. 201-204, Marcel Dekker, New York, NY, USA, 2000.

[24] A. Pozzi and M. Lupo, "The coupling of conduction with laminar natural convection along a flat plate," International Journal of Heat and Mass Transfer, vol. 31, no. 9, pp. 1807-1814, 1988.

[25] M. Char and C. L. Chang, "Effect of wall conduction on natural convection flow of micropolar fluids along a flat plate," International Journal of Heat and Mass Transfer, vol. 40, no. 15, pp. 3641-3652, 1997.

[26] A. V. Kuznetsov and D. A. Nield, "Boundary layer treatment of forced convection over a wedge with an attached porous substrate," Journal of Porous Media, vol. 9, no. 7, pp. 683-694, 2006.

[27] A. J. Chamkha, M. Mujtaba, A. Quadri, and C. Issa, "Thermal radiation effects on MHD forced convection flow adjacent to a non-isothermal wedge in the presence of a heat source or sink," Heat and Mass Transfer, vol. 39, no. 4, pp. 305-312, 2003.

[28] O. Aydin and A. Kaya, "Radiation effect on MHD mixed convection flow about a permeable vertical plate," Heat and Mass Transfer, vol. 45, no. 2, pp. 239-246, 2008.

[29] O. Aydin and A. Kaya, "Non-Darcian forced convection flow of viscous dissipating fluid over a flat plate embedded in a porous medium," Transport in Porous Media, vol. 73, no. 2, pp. 173-186, 2008.

[30] T. Cebeci and P. Bradshaw, Momentum Transfer in Boundary Layers, Hemisphere, Washington DC, USA, 1977.

[31] H. S. Takhar and O. A. Beg, "Effects of transverse magnetic field, prandtl number and reynolds number on non-darcy mixed convective flow of an incompressible viscous fluid past a porous vertical flat plate in a saturated porous medium," International Journal of Energy Research, vol. 21, no. 1, pp. 87100, 1997.

[32] O. Aydin and A. Kaya, "Mixed convection of a viscous dissipating fluid about a vertical flat plate," Applied Mathematical Modelling, vol. 31, no. 5, pp. 843-853, 2007.

[33] C. L. Chang, "Buoyancy and wall conduction effects on forced convection of micropolar fluid flow along a vertical slender hollow circular cylinder," International Journal of Heat and Mass Transfer, vol. 49, no. 25-26, pp. 4932-4942, 2006.

[34] C. L. Chang, "Numerical simulation for natural convection of micropolar fluids flow along slender hollow circular cylinder with wall conduction effect," Communications in Nonlinear Science and Numerical Simulation, vol. 13, no. 3, pp. 624-636, 2008.

[35] M. Kumari and G. Nath, "Radiation effect on mixed convection from a horizontal surface in a porous medium," Mechanics Research Communications, vol. 31, no. 4, pp. 483-491, 2004. 


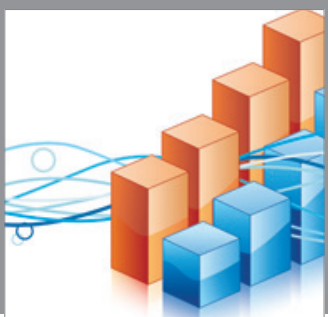

Advances in

Operations Research

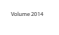

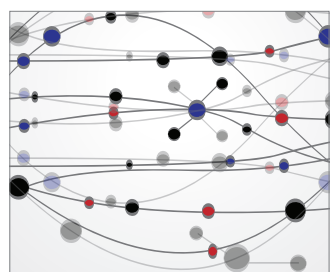

\section{The Scientific} World Journal
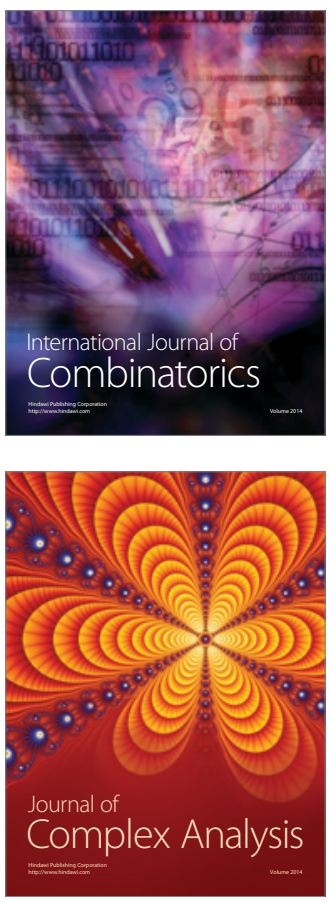

International Journal of

Mathematics and

Mathematical

Sciences
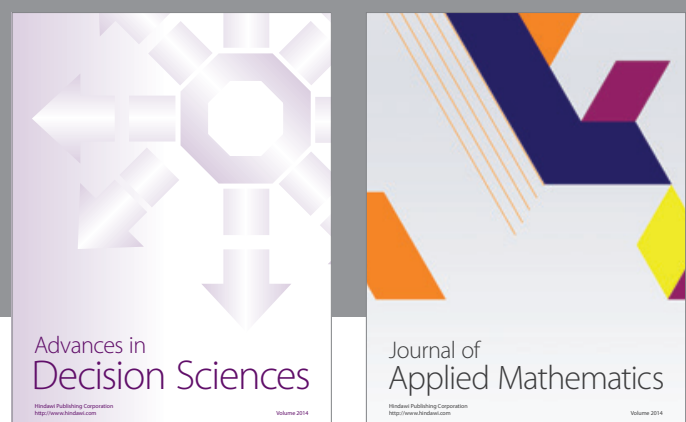

Journal of

Applied Mathematics
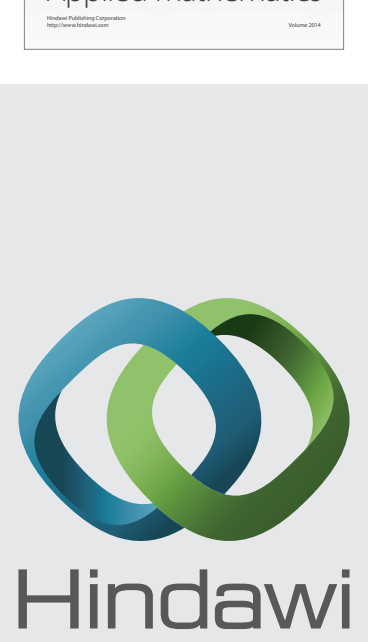

Submit your manuscripts at http://www.hindawi.com
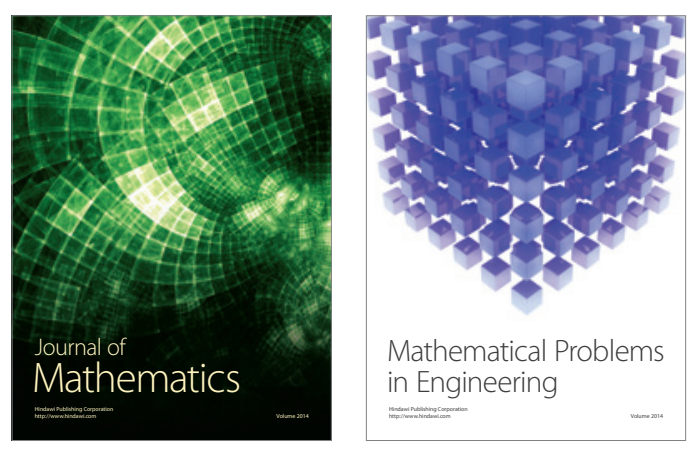

Mathematical Problems in Engineering
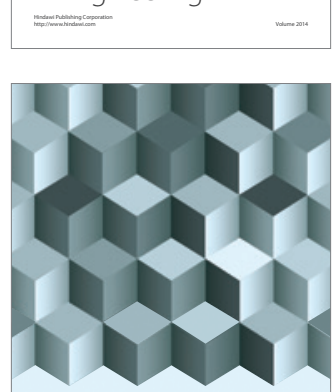

Journal of

Function Spaces
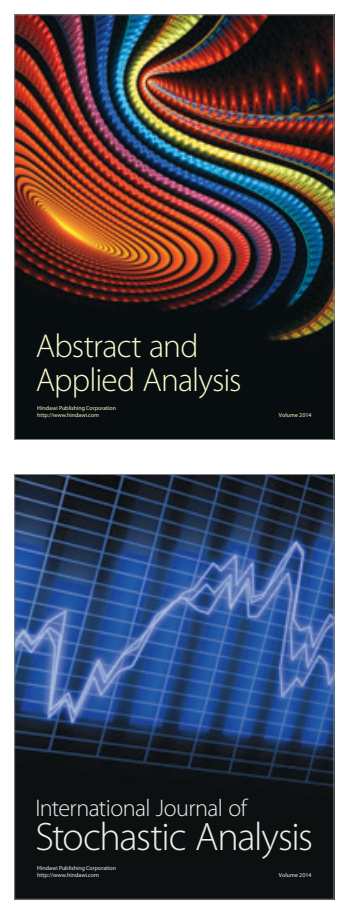

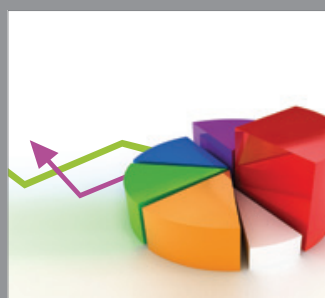

ournal of

Probability and Statistics

Promensencen
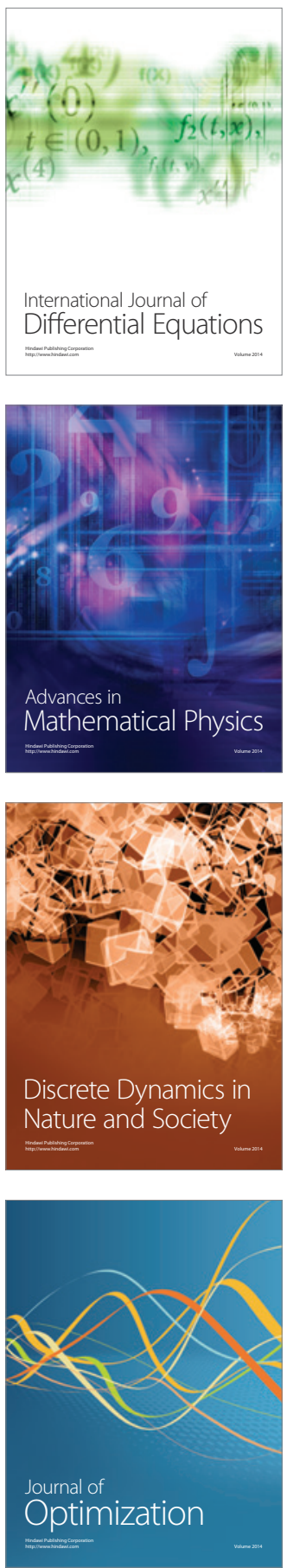